\title{
Bridging the gaps: How students seek disciplinary coherence in introductory physics for life science
}

\author{
Benjamin D. Geller, ${ }^{1}$ Julia Gouvea, ${ }_{5}^{2}$ Benjamin W. Dreyfus, ${ }_{5}^{3}$ Vashti Sawtelle, ${ }^{4}$ \\ Chandra Turpen, ${ }^{5}$ and Edward F. Redish ${ }^{5}$ \\ ${ }^{1}$ Department of Physics and Astronomy, Swarthmore College, Swarthmore, Pennsylvania 19081, USA \\ ${ }^{2}$ Department of Education, Tufts University, Medford, Massachusetts 02155, USA \\ ${ }^{3}$ Department of Physics, George Mason University, Fairfax, Virginia 22030, USA \\ ${ }^{4}$ Department of Physics and Astronomy, Michigan State University, East Lansing, Michigan 48823, USA \\ ${ }^{5}$ Department of Physics, University of Maryland, College Park, Maryland 20742, USA
}

(Received 15 December 2018; published 12 November 2019)

\begin{abstract}
Students in one discipline often receive their scientific training from faculty in other disciplines. As a result of tacit disciplinary differences, especially as implemented in courses at the introductory college level, such students can have difficulty in understanding the nature of the knowledge they are learning in a discipline that they do not identify as their own. We developed a course in introductory physics for life science (IPLS) students that attempts to help them cross disciplinary boundaries. By analyzing student reasoning during recitation sections and interviews, we identified three broad ways in which students in our course meaningfully crossed boundaries: (i) by unpacking biochemical heuristics in terms of underlying physical interactions, (ii) by locating both biochemical and physical concepts within a mathematical bridging expression, and (iii) by coordinating functional and mechanistic explanations for the same biological phenomenon. Drawing on episodes from case-study interviews and in-class problem-solving sessions, we illustrate how each of these types of boundary crossing involves the coordination of students' conceptual and epistemological resources from physics, chemistry, and biology in distinct but complementary ways. Together, these boundary crossing categories form a theoretical framework for classifying student coherence seeking. We explore how the IPLS course helps our life science students fill in the gaps that exist between traditional introductory courses, by finding and exploring questions that might otherwise fall through disciplinary cracks. By identifying these types of explanatory coherence, we hope to suggest ways of inviting life science students to participate in physics and see physics as a tool for making sense of the living world.
\end{abstract}

DOI: 10.1103/PhysRevPhysEducRes.15.020142

\section{INTRODUCTION: IDENTIFYING AND BRIDGING DISCIPLINARY GAPS}

In our interviews with life science students over many years, we have often heard students express the view that the disciplines of biology and physics are distinct, having little to say to each other [1-8]. Physics instructors rarely include significant biology in an introductory physics class, and biology instructors rarely include significant physics in introductory biology classes. Few opportunities exist for life science students to see different disciplinary explanations as meaningfully related, let alone as part of a coherent whole [1-8].

Published by the American Physical Society under the terms of the Creative Commons Attribution 4.0 International license. Further distribution of this work must maintain attribution to the author(s) and the published article's title, journal citation, and DOI.
As a result, life science students encounter disciplinary gaps and inconsistencies as they navigate the undergraduate science curriculum. These gaps promote a disconnected understanding of biological phenomena, with students often developing a fragmented view in which physics contributes little to their appreciation of the natural world, despite the fact that living systems are subject to physical laws and constraints [8]. Students have few opportunities to see how ideas from physics can enhance or expand their understanding of phenomena from chemistry or biology.

One of the primary goals of IPLS courses is to provide students with an opportunity to bridge these disciplinary divides. Rather than rely solely on instructors and curriculum designers to identify these connections, it is important to understand where students see opportunities for increased coordination $[9,10]$. What gaps and inconsistencies do they recognize across the disciplines? Where do they see opportunities to bring together ideas from different disciplinary courses? 
By identifying connections that students see as interesting and relevant to their learning, we hope to suggest a variety of ways of inviting life science students to participate in physics and see physics as useful for making sense of the biological world. Our observations were carried out in the context of an IPLS class specifically developed to attempt to help students make these connections [6,11]. While the class is an introductory physics one, it has biology and chemistry prerequisites. This permits the class to include more significant biological and chemical contexts and examples than is possible in traditional classes.

In this paper we identify three classes of interdisciplinary connections that some of our IPLS students have made. The three classes of connections we identify are not the only possible connections that students might make in an IPLS course, but they exemplify types of interdisciplinary connections that we argue can help students build coherence across the disciplines. Each of these classes coordinates students' conceptual and epistemological resources from physics, chemistry, and biology in distinct but complementary ways. We present these cases as exemplars of the kinds of reasoning that are possible, but often absent from students' university science learning. We do not claim that the cases we describe are representative of the experiences of all IPLS students. Our purpose is to argue that the reasoning illustrated in these cases is desirable and to motivate the need for additional research on how to make these outcomes more common.

Before we describe the connections made by students, we elaborate on the nature of the problem of disciplinary fragmentation and how we have conceptualized coherence across disciplinary boundaries.

\section{A. Explanatory coherence seeking among science students}

Because students' experiences across disciplinary boundaries are frequently disjointed, an important learning goal for IPLS courses is for students to seek coherence among the explanations encountered in their various science courses. Sikorski defines explanatory coherence seeking as the process of "trying to build meaningful, mutually consistent relationships between information" [10]. This coherence seeking is a fundamental feature of learners' sense making, because it allows students to coordinate different perspectives in ways that provide new insight or understanding $[9,12]$.

Whether or not coherence is ultimately achieved, Phillips et al. argue that just being able to identify gaps or inconsistencies is itself a crucial component of scientific practice [13]. Furthermore, identifying these gaps or problems and reconciling them can be exciting and highly motivating for students $[14,15]$. We value coherence seeking for reasons that are similar to why we value a student's attempts to coordinate between conceptual and mathematical expressions. The aim is not to have one subsume the other, but rather for students to be able to understand how various representations are related and to move fluidly among them.

\section{B. Fostering explanatory coherence seeking}

Part of the challenge in fostering interdisciplinary boundary crossing is that different disciplines approach phenomena from different perspectives and ask different questions [9]. These differences are mirrored in the questions and problems that are presented to students in different courses $[2,8,14,15]$. Even when life science students encounter the same phenomenon in different courses, the questions asked about that phenomenon in physics class may be so different in nature from those asked in biology class that students may not see the two discussions as meaningfully related [15].

The differences among disciplines can be exacerbated by instruction that presents students with "school versions" of the disciplines. Physics can seem to be all about solving equations and biology can seem to be all about memorizing terms. These versions present far fewer opportunities to see connections than are present in a scientific community where scientists increasingly work across traditional disciplinary lines. The way in which explanations are constructed in real disciplinary practice can differ significantly from the way the disciplines are described in "school" or in the students' minds.

Consider, for example, the lipid bilayer structure of cell membranes in biology. Biological cell membranes are composed of phospholipid molecules arranged in a particular orientation that minimizes the interaction of the nonpolar hydrocarbon lipid tails with the polar (aqueous) environment of the cell. This phenomenon presents an opportunity to examine the energetic and entropic contributions to the stability of that membrane.

Yet, because of the constraints of typical science curricula, opportunities for interdisciplinary coordination are rare for students. Introductory coursework often presents phenomena from a narrow disciplinary perspective. A biology course might foreground the function of the lipid bilayer membrane in the cell. The spontaneity of its formation or its stability may be mentioned in such a course, but students might still leave the course not viewing these as phenomena in need of explanation. Meanwhile, a typical physics course will discuss energy and entropy, but might not do so in a way that is easily applicable to biological phenomena. As a result, students may not appreciate the potential for coordination between disciplines in the way that we would hope.

Our interest is in supporting students in seeking coherence in their own learning. An important feature of many of the tasks in our IPLS course is an exploration of biological phenomena from multiple disciplinary perspectives. As we describe by way of examples throughout this paper, explanatory coherence in the context of our course refers to connecting ideas from these multiple disciplinary pathways. 


\section{Coordination does not mean unification}

Some philosophers of science have argued that an essential feature of explanation is the unification of seemingly disconnected phenomena in order to achieve some degree of global rather than local explanatory power $[16,17]$. Others have argued that local explanations cannot be neatly subsumed by global theories $[18,19]$. While we appreciate the desire for unification in some instances, we have not found this framework to be particularly useful in the classroom. Instead, we have found frameworks that preserve contextual differences to be productive for fostering connections across disciplines because they preserve the disciplinary utility of different explanatory approaches.

Rather than a search for a global unity, contextpreserving approaches seek coherence among local perspectives. In particular, we argue that coherence can arise in the context of a single phenomenon, when different explanations for that phenomenon are coordinated across disciplinary boundaries.

To make this notion of coordinating multiple explanatory pathways concrete, consider again the lipid bilayer structure of cell membranes. Different disciplinary courses may decompose this phenomenon in different ways to address different questions. ${ }^{1}$

A biology course might point to the importance of the polar and nonpolar portions of the membrane in facilitating particular cell functions such as intercellular transport and signaling.

A chemistry course may not discuss cellular functions at all, but may instead describe bilayer formation in terms of Gibbs free energy [20,21], using a negative change in the free energy of the system to stress that the spontaneity of lipid bilayer formation [22]. In turn, this negative change in free energy can be understood as resulting from the interplay of the energetic and entropic terms in the free energy expression $G=H-T S$. $^{2}$

A physics course, if it involves a molecular-level discussion of lipid bilayer membranes at all, might analyze the electrostatic interactions between individual lipid and water molecules, and of the degrees of freedom available to those molecules. ${ }^{3}$

Each of these approaches to describing the bilayer membrane has value within the disciplines. They are also

\footnotetext{
${ }^{1}$ We recognize that these are not the only questions such courses may be interested in answering; we provide these examples only to establish why students might find the different presentations disjointed. In Sec. II, we describe how the assigning of disciplinary labels to particular explanatory moves was driven by the student's own statements.

${ }^{2}$ Gibbs free energy is particularly useful in biology and chemistry because the temperature and pressure are often considered constant during biological processes and chemical reactions.

${ }^{3}$ Chemistry courses might also describe the spontaneous formation of lipid bilayers in terms of a balance between enthalpy and entropy, but rarely would such courses delve into the fundamental forces responsible for the values of these terms.
}

interconnected: molecular interactions provide a mechanism for spontaneity; spontaneity provides a way to understand the stability of the membrane as a chemical structure; and cellular functions give biological meaning to the particular molecular structure that forms.

This characterization of different disciplinary treatments of the cell membrane is intentionally simplistic. We recognize that biology courses do not only ask structurefunction questions, chemistry courses do not only describe the spontaneity of processes in terms of Gibbs free energy, and physics courses do not always go into detail at the molecular level. Still, the disciplinary framework that students encounter can be viewed as a resource for coherence seeking. By creating opportunities for students to consider ideas from multiple courses at the same time, they themselves might identify and make connections as they seek to build their own sense of coherence. If a physics course does not bring in examples (like the bilayer membrane) of biological and chemical importance, there is little opportunity for the explanations encountered in physics class to be coordinated with those in other courses. A primary goal of our IPLS course was to create space for such coordination.

Our purpose in characterizing explanatory frameworks in this way is not to comprehensively represent the complex interdisciplinary network of ideas related to any natural phenomenon, but rather to identify a set of prominent guideposts defining the distinct disciplinary perspectives that our students encounter in their introductory science coursework. These guideposts help us to identify classes of interdisciplinary connections made by students in our IPLS course, and to identify the gaps that students find it especially satisfying to bridge.

Beginning in Sec. III, we identify three classes of interdisciplinary connections, three broad ways in which our students have formed meaningful interdisciplinary links in IPLS:

- Connecting biochemical heuristics to a physical picture of microscopic interactions.

- Connecting physical and biochemical concepts through bridging equations.

- Connecting mechanistic and functional explanations for biological phenomena.

By categorizing these connections, we hope to point out multiple ways in which productive interdisciplinary coherence seeking can happen. These categories represent distinct but complementary ways in which students coordinate disciplinary concepts and epistemologies in IPLS. Before fleshing out that discussion, we examine the process by which this categorization was established.

\section{METHODOLOGY}

All data in this paper are drawn from the IPLS course (NEXUS/Physics) at the University of Maryland, College Park (UMCP) $[6,11]$. We draw primarily on two sources of 
student data: interviews conducted with students over the two-semester course, and video of students working together in small groups on weekly problem-solving tasks. As described in more detail below, our approach was to identify and characterize promising instances of coherence seeking among the large set of qualitative data we obtained.

Because a year of biology and a semester of chemistry were prerequisites for the course, the students in our study were mostly juniors (with a few sophomores and seniors) majoring in biology or a related life science discipline. Many of the students hoped to pursue medical school or another health profession after graduation. Although just over half of the students at the UMCP campus are not white, the students in our study were predominantly white.

Thirty-one students were enrolled in the year-long IPLS course during year one of our study. Twenty-two (approximately hour-long) interviews were conducted with 11 of these 31 students. Thirty-one students were enrolled in year two of our study, during which a total of 26 (also hour-long) interviews were conducted with 12 of the 31 students. In all, the 23 students who were interviewed a total of 48 times represent more than $1 / 3$ of the students enrolled in the course over two years. In addition to these interviews, small group problem-solving sessions were filmed weekly throughout each semester of IPLS.

Interviews conducted in IPLS served multiple purposes. In order to get a sense for how our students had been exposed to concepts like energy and entropy in prior biology and chemistry coursework, we interviewed students at the start of the course about their previous interactions with these topics. Because the purpose of these initial interviews was to establish which ideas students were most likely to bring up spontaneously when presented with specific contexts that they had not yet encountered in the IPLS course itself, these initial interviews about energy and entropy were highly structured. Each student was shown schematics of three particular physical processes (the expansion of gas molecules to fill a container when a dividing partition was removed, the equilibration of two objects initially at different temperatures, and the formation of a cell membrane from individual lipid molecules) and asked to describe the processes shown. They were also asked to describe why the process generally only proceeded spontaneously in one direction. The results of just these initial interviews have been reported elsewhere [20] and provided a jumping off point for the more flexibly designed interviews that followed later in the year.

As the year progressed, interviews provided an opportunity for students to further articulate and expand upon reasoning that we observed in lecture, on exams, or in problem-solving sessions. By this point, students had been exposed to ideas about energy and entropy in the IPLS setting, and our goal was to probe how they were engaging with these ideas. As individual students engaged differently with different examples, a more flexible interview protocol was implemented in these later interviews. Students were given space to describe moments in the course that stood out to them as particularly meaningful. In order to elicit evidence of coherence seeking, each interviewee was asked to describe any moments in the course where they saw meaningful connections between ideas in IPLS and their other prior or current coursework. When students did describe such moments, the interviewer would sometimes interject more directly in order to elicit more nuanced reasoning or to unpack the student's thought process. This was essential, since the goal was to identify the ways in which our IPLS students were seeking coherence, not just to identify the list of topical areas that students felt were most connected to their other coursework. The interviews were not designed to test what the students knew, but to determine what resources they had available and could employ when processing examples encountered in the course. As such, some interviews involved a minimal amount of "teaching," in order to further the conversation and see how the student responded.

The weekly problem-solving (recitation) sections provided an opportunity for students to work in groups of four on problems that explored rich biological contexts. Each recitation section was facilitated by one teaching assistant (TA) and one or more undergraduate learning assistants (LA) [23]. These sections were one of the best opportunities in the course for students to explore the relationship between physical models and biological phenomena. Recitation tasks were designed with specific intent to draw on students' ideas from biology and chemistry, often with the goal of reconciling those ideas with newly learned principles of physics. Because students were working in groups with minimal intervention on the part of the TAs or LAs, recitation section data provided us with a more unfiltered view of student reasoning than was possible in the interview setting.

The interviews and problem-solving sessions produced a wealth of student data. At least two members of our research team independently reviewed all the recitation and interview transcripts, and each researcher identified any instances where students appeared to be meaningfully engaged in coherence seeking. The two researchers then presented the most promising instances to the entire research team, and they were reviewed by all the authors. Since our goal was to identify some (but not all) possible bridging approaches, we looked for and identified patterns that reappeared. We considered an approach to disciplinary gap bridging to be nonunique and reappearing when (a) it was exhibited by multiple students, and (b) it was exhibited in both the interview and recitation settings. In the end, of the 23 students interviewed during the study, more than half explicitly referenced episodes that would exemplify one or more of the classes of connections identified. 
Having identified the bridging approaches that were exhibited by multiple students, and across both interview and recitation data, a select number of episodes were chosen for inclusion in this paper. The particular episodes included were chosen because they represent instances in which students make meaningful progress (or describe having made meaningful progress) in interdisciplinary sense making. This progress was most often accompanied by noticeable affect, by a sense of resolution or satisfaction expressed by the students in having bridged a disciplinary divide. As researchers, this affect served as a useful indicator that the episode was worth exploring in even greater detail. Our goal was then to unpack the conceptual and epistemological sources of the observed affect, to understand and classify the nature of the interdisciplinary connection that was meaningful to our students. In Sec. VI we describe how the examples that we have selected are not unique. They illustrate categories of interdisciplinary sense making that we have observed students making throughout multiple iterations of our IPLS course. This does not indicate that our categories are representative of all students in our course, and certainly not of IPLS students generally. Rather, our goal in defining these categories is to provide structure to the ways that we observe our students interacting meaningfully with examples in IPLS, and to provide a framework of analysis for future work.

Throughout our analysis the locating of ideas or claims within particular disciplines is motivated primarily by our IPLS case-study students' own words. These students see certain ideas as coming from biology class, and others as coming from physics class. Often we see evidence that these assignments are closely tied to a student's disciplinary epistemology, his or her sense of what it means to do biology or physics. Wherever connections are identified as interdisciplinary in the analysis that follows, it is because the students themselves have tagged the ideas they are coordinating with distinct disciplinary labels. When students describe connecting ideas from biology with ideas from physics, for example, that connection is treated in this analysis as an interdisciplinary one, regardless of whether we as researchers might quibble about the accuracy of the student-chosen disciplinary labels.

We now consider the three classes of interdisciplinary connections that students made in our course, those listed in bullet points at the end of Sec. I. We present these three classes in Secs. III-V by way of examples selected to illustrate their value in concrete contexts. After establishing the nature of the three classes of connections, we argue in Sec. VI that they span the landscape of interdisciplinary connections that we have observed in our IPLS course.

\section{CONNECTING BIOCHEMICAL HEURISTICS TO A PHYSICAL PICTURE OF MICROSCOPIC INTERACTIONS}

One goal of our IPLS course is to unpack the physical mechanisms underlying biological phenomena that are only described phenomenologically in typical introductory biology and chemistry courses. An example of this is the diffusion of particles or gases as a result of a concentration gradient, a phenomenon with which life science students become familiar but for which they are often not provided a mechanistic explanation in their introductory biology or chemistry courses $[24,25]$.

The discussion of diffusion in our IPLS course emerged from a curricular thread focused on the relationship between random and directed motion. In particular, a recitation activity on 1D random walks afforded students the opportunity to prove that a localized group of particles moving in random directions tends to spread out to fill the space available to it. The task asked students to make sense of the tendency for particles to go from high to low concentrations in terms of the collisions with nearby particles and a minimal amount of probabilistic reasoning. Students were prompted to extrapolate from the 1D diffusion example in order to prove that macrostates associated with a greater number of microstates are more likely, and to associate entropy with this measure. The spontaneous increase in entropy is seen as arising not just from an abstract "counting" of available states (as is emphasized in a traditional coin-flipping example), but from a physical mechanism: real microscopic physical interactions leading to a meaningful physical result.

Diffusive (passive) transport plays an essential role in numerous biological processes [26,27], but introductory biology and chemistry courses rarely have the time to devote to unpacking the molecular mechanism for such diffusive motion. Gavin, a case study student in our IPLS course, found the unpacking of diffusion in mechanistic terms to be highly satisfying and referenced this example in describing the role that IPLS played in his education more generally:

(1) Gavin: This [IPLS] class was very good about telling us about thermodynamics and entropy's role in the universe... And I think diffusion was when everything started to click; when we talked about how molecules go from higher concentration to lower concentration because they're bumping into each other so much, and so these Newtonian interactions were able to move particles away from one another... there was less collisions and stuff like that... And so I felt like that's when things started to click (snaps fingers) ... I was like that's why molecules go from higher concentration to lower concentration...

(2) Interviewer: So you already knew that it happened?

(3) Gavin: I knew that it happened but then I was like how the hell do they know where the lower concentration is?! And in biology we never explain that (brushes arm across his chest). And I think that biology has done obviously very brilliant things and I love biology, but as far as the professors, they're very knowledgeable but they have to go over so 
much stuff that they don't really take time to explain why things happen. And I'm a very "why" kind of person; I want to understand why does this happen? And that's why I struggle with [organic chemistry] so much, because it's like 'memorize the mechanisms and take the test' (throws up his hands) ... well how the hell do I know why the mechanism is happening in the first place?!

(4) Interviewer: How do the molecules know what to do...

(5) Gavin: Exactly. And why do they do this bouncing thing (moves hands back and forth) and it was never explained to me very well, and then when I take this [IPLS] class and understand, oh, this is why molecules interact the way they do, this is why you are going to have this expansion of particles over space.

(6) Interviewer: Yeah.

(7) Gavin: It's because they collide less often when they're further apart than when they're together. And they are going to want the least colliding orientation which is going to have the most microstates which is therefore going to have the greatest entropy.

(8) Interviewer: So it connected... you knew that it wanted the greatest entropy, and it connected sort of underneath it what was causing?

(9) Gavin: Right it gave me a foundation...

(10) Interviewer: And that was satisfying to you?

(11) Gavin: That was very satisfying... understanding the why really gave me the confidence in order to go into tests and be able to rationalize why things work the way they do and what to look for.

Although Gavin is not entirely "correct" in his description of the physical mechanism underlying diffusion, ${ }^{4}$ it is clear that he senses that such a description is possible. This exchange illustrates the nature of the interdisciplinary connection that Gavin has made, as well as his satisfaction in having made it. Unpacking heuristic statements like "particles know to go from high to low concentration" in terms of the underlying physical interactions between individual particles puts Gavin's overall understanding of diffusion on a more solid (better integrated) foundation.

Gavin identified the heuristic about particles going from high to low concentration as one that came from his biology and chemistry courses, and he identified the unpacking of diffusion in terms of collisions with nearby molecules as happening in physics class.

As detailed in Sec. I, this matching of particular ideas with particular disciplines is not meant to capture the complex interdisciplinary network of ideas related to diffusion, but rather to identify a set of prominent

\footnotetext{
${ }^{4}$ Gavin has misidentified the collisions among the molecules of interest rather than the collisions with the surrounding fluid (thermal bath) as responsible for the random walk of the diffusing molecules.
}

guideposts defining the disciplinary perspectives that our students encounter and describe. Because the IPLS course asks questions about the physical mechanisms underlying diffusion that neither introductory biology and chemistry courses nor traditional introductory physics courses typically ask, the course bridges a gap that might not otherwise have been bridged.

In turn (3) of the interview exchange, Gavin reflects on his experience in biology and (organic) chemistry courses. He suggests that, for reasons having to do with pressure to cover a large amount of material ("they have to go over so much stuff"), his biology and organic chemistry courses have not devoted time on the explication of "why things happen." In this context, Gavin's "why" questions would perhaps better be interpreted as "how" questions, since it is not clear that an evolutionary answer to "why particles diffuse" would elicit for Gavin the same satisfaction that we see in the above exchange. ${ }^{5}$

The difference between Gavin's epistemological orientations toward biology and physics in this moment is apparent, and is largely responsible for his seeing the causal mechanistic account of diffusion as being a "physics" account. Gavin's epistemological orientation toward biology in this moment is one in which he sees the discipline as failing to take up mechanistic explanations of the sort that IPLS provides for diffusion (or perhaps even failing to ask questions for which a mechanistic answer is appropriate). His arm movements and tone both convey frustration with such a misalignment. On the other hand, where Gavin's epistemological orientation toward biology is in tension with his identification as a "why kind of person," his epistemological view of physics as a place where sense making happens aligns with this identity in such a way that his words suggest an affective response to physics that is notably more positive [25]. He describes being "very satisfied" by the explanation in physics class and feeling more "confident." We return to these affective issues in Sec. VII C.

The Gavin episode points to one way in which heuristic biochemical statements about emergent phenomena can be coordinated with fundamental physical principles. Namely, the phenomena described by the heuristic can be seen as directly emerging from a detailed analysis of the interactions of the microscopic particles involved in the process. Gavin develops a physical picture for how individual molecules bump into each other, and how such random collisions between molecules can generate directed bulk motion.

\footnotetext{
${ }^{5}$ Indeed, in an interview conducted with Gavin several months after the conclusion of the IPLS course, Gavin reiterates that being able to run a simulation of the diffusive process in his mind, being able to picture the intermolecular collisions between diffusing particles, is tied up with his sense that he "understands" diffusion.
} 


\section{CONNECTING PHYSICAL AND BIOCHEMICAL CONCEPTS THROUGH BRIDGING EQUATIONS}

For processes in which intermolecular interactions cannot be ignored (which is most of them), detailed mechanistic accounts of the motions of individual particles are impractical. Nevertheless, we observe students making meaningful interdisciplinary connections between biochemical heuristics and fundamental physical mechanisms in these cases as well.

When detailed physical accounts are not possible (nor practical), we observe mathematical relationships playing a central role in students' sense that their understanding from different disciplines is related and coherent. In particular, in our IPLS course the Gibbs free energy relationship $\Delta G=$ $\Delta H-T \Delta S$ becomes an essential bridge between the spontaneity of processes (as indicated by a negative change in the Gibbs free energy $G$ ) and the physical factors that determine the enthalpy $H$ and entropy $S$. The Gibbs free energy relationship is used throughout introductory biology and chemistry courses, but is rarely discussed at all in standard introductory physics courses [20].

As we discuss in Sec. VI, some mathematical relationships are better than others in their capacity to bridge physical and biological ideas. The Gibbs free energy relationship is a particularly useful link between fundamental physical and biochemical ideas. In this section we explore two examples where students make meaningful interdisciplinary connections by examining the Gibbs free energy relationship through the lens of our IPLS course.

\section{A. Using the Gibbs free energy expression $\Delta G=\Delta H-T \Delta S$ to unpack the "like dissolves like" heuristic}

The observation that oil and water do not mix is identified in many introductory biology and chemistry courses as an example of the widely stated heuristic, "like dissolves like" [21]. This rule of thumb refers to the idea that solutes dissolve in solvents only when both solute and solvent molecules exhibit similar chemical polarities. Polar compounds and ionic compounds like table salt dissolve in polar substances like water quite easily, while nonpolar carbon chains like oil do not. Thus like dissolves like is frequently used as a heuristic rule to predict that oil and water do not mix. It is also used to describe the formation of lipid bilayer cell membranes, as mentioned earlier in this paper.

What such rules do not do, and are not designed to do, is to ground phenomenological predictions in mechanistic reasoning or foundational laws of nature. Indeed, heuristic rules can sometimes seem to be superficially at odds with more general physical principles. How, for example, does one reconcile like dissolves like with the universally accepted principle that opposite electrical charges attract?
The phrase like dissolves like by itself does not explicate the underlying physical explanation for why it holds, nor does it specify the conditions for when it is appropriate to use it.

For molecules of equal size, students learn that the electrostatic interaction between a polar molecule like water and a nonpolar molecule like oil is stronger than the electrostatic interaction between two oil molecules. ${ }^{6}$ As a result, electrostatic energy is lower when a nonpolar molecule interacts with a polar molecule than it is when it interacts with another nonpolar molecule. But this is an energetic effect, and the spontaneity of processes in the natural world is determined not by whether the energy is lowered during the process, but rather by whether free energy is lowered. This key distinction, a discussion of which is rarely found in either introductory biology or introductory physics courses, is at the heart of any effort to reconcile the formal principle "opposite electric charges attract" with the informal principle like dissolves like. The conceptual bridge between energy and free energy is entropy. When one considers the entropic effects at play in the oil and water example, it becomes clear that the hydrophobic effect is in fact entropically driven [21].

Like dissolves like is a thermodynamic rule of thumb that can be understood in terms of the Gibbs free energy $G=H-T S$, while opposite charges attract is a fundamental electrostatics principle. Since students are not often asked to discuss electrostatics and thermodynamics at the same time [21], and often not even in the same course (for example, electrostatics is typically a second-semester introductory physics topic, whereas thermodynamics is often covered in the first semester if it is covered at all), the two ideas are not easily reconciled in practice.

Elena, a student in our IPLS course, was interviewed about her understanding of the oil and water phenomenon. Recalling that the electrostatic attraction of a nonpolar molecule to a polar molecule was greater than the electrostatic attraction between two nonpolar molecules, Elena struggled to understand the spontaneous separation of oil and water in a way that appealed to the electrostatic forces that she felt were responsible. She knew that like dissolves like, as she put it, but was unsure how to connect the heuristic with her knowledge of the relative strengths of intermolecular electrostatic interactions. Cleverly, she proposed that perhaps it was the greater length of the oil molecule in comparison to the water molecule that was responsible, so that while the pound-for-pound interaction

\footnotetext{
${ }^{6}$ To be clear, it is not always the case that interactions between nonpolar molecules (van der Waals interactions) are weaker than those between nonpolar molecules and water molecules. Depending on the nature of the specific molecules involved (and in particular on their size), it is possible for van der Waals interactions to dominate. Students, however, may not be made aware of this subtlety when learning about the relative electrostatic strengths of various interactions.
} 
strength between nonpolar molecules might be weaker, the overall interaction between long oil molecules was sufficient to break up the water-oil interactions. When the interviewer points out that the water molecules might form a line having the same length as an oil molecule, Elena is troubled by the apparent inconsistency between her electrostatic knowledge and what she knows to be observably true:

(1) Interviewer: ... And if the numbers came out that the water line was more attracted to the hydrocarbon line than two hydrocarbon lines were, would that bother you?

(2) Elena: That would bother me.

(3) Interviewer: Why would that bother you?

(4) Elena: Because then to me it would just seem like, well, why wouldn't water completely surround each individual hydrocarbon, if it overall has the stronger interaction than the hydrocarbon with the hydrocarbon?

Unable to press on using electrostatics alone, the interviewer points to an equation on the board relating free energy, enthalpy, and entropy $(\Delta G=\Delta H-T \Delta S)$ and asks Elena to incorporate that relationship into her story. ${ }^{7}$

(5) Interviewer: So in terms of this (points to $\Delta G=$ $\Delta H-T \Delta S$ equation on whiteboard), what would the story be if the line of water is attracted more to the line of hydrocarbons [than are two hydrocarbon lines]?

(6) Elena: Ok, so, now this is where I kind of have two separate thoughts. Here (points toward the page with the oil and water task) we are talking about like electrostatic interactions...

(7) Interviewer: Where do those go (looking toward the board)?

(8) Elena: I just don't feel like they're involved in there (circling the equation with her hand) at all! So that's why I'm kind of having trouble piecing the two together in my mind.

(9) Interviewer: OK, gotcha.

(10) Elena: And I think that's also why that [quiz] question really confused me when [the professors] brought up electrostatic interactions. Like I'm thinking entropy (points toward $\Delta G=\Delta H-T \Delta S$ equation on whiteboard) and you're thinking electrostatic interactions! How do those come together?

When asked to unpack the Gibbs free energy equation, and in particular the meaning of the $\Delta H$ term, Elena actually does herself uncover the fact that electrostatic interactions are buried inside.

(11) Interviewer: OK, but like what factors helped you to think about whether [the change in internal energy]

\footnotetext{
${ }^{7}$ As noted in the Sec. II, we were not trying to test what the student knew, but rather trying to determine what resources she had available and could employ in her processing of new information. This was an instance where the interview involved a small amount of "teaching" in order to elicit this information.
}

was positive or negative? Like what were you thinking about to determine $\Delta H$ for the process of oil and water?

(12) Elena: Internal energy.

(13) Interviewer: And what did that... how did you figure out what sign that had?

(14) Elena: We were thinking, well is the internal energy changing? (thinks about it...) I honestly don't remember what we said... I feel like it was... positive? ... Well, ok, so if you have interactions, you have bonds and you're breaking bonds and reforming them... I think that's where we got it from.

(15) Interviewer: OK.

(16) Elena: So actually I guess the interactions, they're electrostatic interactions, so now it makes sense. (Laughs).

When she reasons that the intermolecular electrostatic interactions involved in bond breaking and bond formation help to determine the value of $\Delta H$, and thereby inform the overall spontaneity signified by the sign of $\Delta G$, Elena proclaims with relief that things have come together for her. She is also then in a position to understand how it is possible for free energy to be lowered when oil and water separate even if the energetics alone were to suggest otherwise.

(17) Interviewer: So, going back to... if we somehow looked up the numbers and found that a chain of waters was more attracted to a hydrocarbon chain than two hydrocarbon chains were [to each other], could you still tell the oil and water story?

(18) Elena: I think so, because you would have a positive $\Delta H$ here [for oil and water separating], but as long as the entropy (points to $\Delta S$ term on board) was higher and this (points to $\Delta S$ term) kind of overwhelms this (points to $\Delta H$ term), as long as it wasn't too much of a [positive $\Delta H$ ], you would still have a negative $\Delta G \ldots$ I feel like I can explain this so much better than I could have last semester. (Laughs).

In the course of completing and reflecting on the oil and water task, Elena becomes aware that the energetic and free energetic realms are not connected for her like she would like them to be. She struggles to find a place for her energetic knowledge about electrostatic attraction within the context of an equation that she associates with thermodynamic and free energy considerations. During the interview, however, Elena uses her understanding of how the Gibbs free energy depends on enthalpic effects to begin the reconciliation process. By situating electrostatics within the enthalpy term of the Gibbs free energy expression, she connects for herself two realms that were previously distinct and unrelated, the "two separate thoughts" that she references in turn (6).

At the start of this episode, Elena is able to answer the question of why oil and water separate by referring to the phrase like dissolves like. What she would have struggled 
to do successfully is to answer the question, "how do oil and water separate?" Questions such as how do oil and water separate? were black boxed in her prior biology and chemistry coursework to the extent that she was not aware of any contradiction in her thinking. By confronting a task that asked her to at once consider both her thermodynamic observations and her understanding of electrostatic physical principles, it was evident to her that boxes needed to be opened.

Much as Gavin expresses frustration in not being able to account for why particles move in the manner that they do during diffusion, Elena is initially bothered by her inability to account for the spontaneous separation of oil and water in terms of intermolecular interactions. For Gavin, resolution involves a description of Newtonian molecular collisions and how those collisions result in the spreading of particles. For Elena, resolution involves locating the competing effects of energy and entropy inside the Gibbs free energy relationship and unpacking how that competition results in spontaneous behavior. This resolution allows Elena to say that "now it makes sense." We return to this comparison between the Gavin and Elena examples, and how both can be seen as worthwhile types of mechanistic accounts of biological phenomena, in Sec. VII B.

Elena has found a way to locate her conceptual understanding of electrostatics within the expression that determines spontaneity in biochemical processes $(G=H-T S)$. In this way, it is the mathematical relationship between free energy, enthalpy, and entropy that plays a central role in Elena's interdisciplinary bridging. She associates the sign of $\Delta G$ with the spontaneity expressed by the biochemical heuristic and identifies the internal energy within the enthalpy term as dependent on the relative strengths of intermolecular electrostatic interactions. The mathematical relationship provides a location for bringing together the "two separate thoughts" that Elena says were previously uncoordinated.

\section{B. Using the Gibbs free energy expression $G=H-T S$ to understand "work" in the context of enzymatic catalysis}

Violet, another student in our course [28], uses the free energy expression as a bridge between her physical and biological conceptual resources for thinking about how enzymes function. In particular, Violet finds the IPLS course helpful in making sense of "work," a term she had encountered in the context of enzymatic catalysis in her biology courses.

Enzymes catalyze (speed up) reactions by lowering the activation energy, often by bringing particular substrates into closer proximity or by positioning substrates into a particular orientation. Enzymes do not change the overall free energy change during a biological process, but they do catalyze such processes. As such, they alter the shape of the curve representing free energy as a function of reaction coordinate, even if they don't change the distance between the initial and final states on such a curve [23].

In an interview setting, Violet describes the bridging role the free energy relationship played in helping her to describe enzymatic activity:

(1) Violet: You get this equation $[G=H-T S]$ in chemistry and biology and now in this class. But you learn it from all different ways, all different angles, and I feel like in this class it's so much combined with biology that you have to put those two realms together... because in biology $\Delta G$ is how much free energy you have to do work in the system. And now in this class you actually have a specific definition of what work actually is, and instead of just like 'oh it can make this product,' you can see how an enzyme fits into what work is... I mean we haven't touched on that [in this course] but it's like, [when enzymes are] changing configurations... [that process] needs a force, so it coincides with it...

(2) Interviewer: And so enzymes are connected with this equation in biology?

(3) Violet: Yeah, I mean overall an enzyme is used to catalyze a specific reaction, and that specific reaction will have a $\Delta G$ that corresponds with it...

Violet notices that the enthalpy term in the free energy expression contains within it the $p \Delta V$ work done by the system $(\Delta H=\Delta U+p \Delta V)$, and associates that $p \Delta V$ work with a force acting over a distance. Violet goes on to clarify how this definition of mechanical work helps her to make sense of enzymatic catalysis:

(4) Interviewer: Now you feel like you have a more specific understanding of what? Work...?

(5) Violet: Exactly. Because, when the enzymes come together, and they bring the products and the substrates together, they're interacting as that force that will like shove the two things together...

(6) Interviewer: OK.

(7) Violet: So the reaction does occur more fast, and the enzyme, like in order to become active, needs to usually change its conformation... which can be equivalent to changing a displacement.

In turn (7), Violet makes a key connection between macromolecular conformational changes described in biology and chemistry courses and the general idea of displacement as described in IPLS. This connection allows her to relate the spontaneity of biological processes like enzymatic catalysis to fundamental definitions of mechanical work and makes the physical underpinnings of the free energy expression relevant to her description of how catalysis happens.

Violet goes on to describe how enzymes catalyze chemical reactions by lowering the activation barrier in a free energy plot. She knows that enzymes accomplish this by bringing into contact various substrates involved in the 
reaction, but until identifying work as "force over distance" 8 she cannot coordinate the idea that enzymes "shove things together" with the idea that enzymes lower the activation barrier. Connecting these ideas requires both an association between free energy and work (an association that Violet makes by unpacking the free energy expression), and an association between work and the application of force over distance.

Violet identifies the notion of work as "force over a distance" as a physics idea and identifies what she knows about enzymes and their function as coming from biology and chemistry. She coordinates these concepts through the free energy relationship in a way similar to Elena's use of the same expression to connect electrostatic interactions to the spontaneous formation of lipid bilayers. Where Elena locates electrostatic forces within the enthalpy term in the free energy relationship, Violet identifies forces (and the work they do) more broadly within that same term. Both the Elena and Violet examples point to the value in carefully framing the free energy relationship as an interdisciplinary bridge between physical and biological ideas.

Lipid bilayer formation and enzymatic catalysis are both "messy" biological processes for which detailed mechanistic accounts of the motions of individual particles are impractical. Nevertheless, we observe both Elena and Violet making meaningful interdisciplinary connections. Both Elena and Violet use the mathematical expression for Gibbs free energy to connect ideas from different disciplinary courses. The expression serves as a link between the spontaneity of biological processes (lipid bilayer formation and enzymatic catalysis, respectively), as represented by the sign of $\Delta G$, and the physical factors that determine the enthalpy change $\Delta H$ during such processes (intermolecular electrostatic interactions and mechanical work, respectively).

\section{CONNECTING MECHANISTIC AND FUNCTIONAL EXPLANATIONS FOR BIOLOGICAL PHENOMENA}

The Elena episode in Sec. IVA points to one way in which students might coordinate disciplinary ideas about a biological phenomenon like bilayer formation. In reflecting on the bilayer problem in an interview, Elena uses the expression for Gibbs free energy to link her understanding of biochemical spontaneity to what she knows to be true about the relative strengths of electrostatic interactions. A recitation exchange between two other IPLS students, Hollis and Cindy, illustrates a second way in which the same biological context offers an opportunity for a different kind of interdisciplinary coordination.

\footnotetext{
${ }^{8}$ From context not presented, it is apparent that Violet means here "a force acting over a distance," and therefore "force times distance." She does not literally mean "force over distance, i.e., force divided by distance."
}

Having worked through a two-week recitation exploring the qualitative role of energy and entropy in determining biological structure formation [20], the students were asked to consider why it is that lipids self-arrange into bilayer membranes rather than monolayers. Hollis attempted to position her group's physical explanation for bilayer stability alongside her own biological knowledge about the biological function of such structures. She expressed to Cindy that she was not sure that her physics understanding was consistent with her understanding of cell biology:

(1) Hollis: I mean, in terms of biology and biochemistry, the reason why it forms a bilayer is because polar molecules need to get from the outside to the inside [of the cell], so you need a polar environment inside the cell. But I don't know how that makes sense in terms of physics...

With Cindy's help, Hollis becomes satisfied that the explanation they have been working together to construct is in fact consistent with her expectation from biology:

(2) Cindy: So what I'm saying is... if [the hydrocarbon tail] is hydrophobic and interacting with water, then it's going to create a positive Gibbs free energy, so it won't be spontaneous. So, in this [monolayer] case you have the hydrophobic tails interacting with whatever's on the inside of the cell, which is mostly water, right?

(3) Hollis: Or other polar molecules.

(4) Cindy: Yeah, other polar molecules... and that's bad.

(5) Hollis: And that's why...OK.

(6) Cindy: That's a positive Gibbs free energy.

(7) Hollis: Yes. See, you explained it perfectly!

After writing for a few moments, Hollis reaffirms her satisfaction in having arrived at a "physics" explanation alongside her "biology" explanation:

(8) Hollis: So that made perfect sense, the way you said it.

(9) Cindy: OK.

(10) Hollis: Because I was thinking that, but I wasn't thinking it in terms of physics. And you said it in terms of physics, so it matched with biology (fist pump).

(11) Cindy: Good.

Hollis' fist pump at the end of turn (10) conveys her feeling of resolution in having co-constructed (along with her groupmates) parallel physical and biological explanations for lipid bilayer formation.

This functional role of compartmentalization and membrane transport is, for Hollis, the "biology explanation" for cell membrane formation. She says that lipid bilayers spontaneously self-assemble because such structures form semi-permeable barriers through which only certain molecules can pass. As Elena does, Hollis and her group associate the spontaneous formation of lipid bilayers with a negative change in free energy. Over the course of the recitation, they unpack the condition for spontaneity in 
terms of its energetic and entropic contributions. The explanation that results from the unpacking is, for Hollis, the "physics explanation."

In reality, Hollis' biology and physics explanations do not stem from the same question. Her biology explanation in terms of cellular compartmentalization is functional, providing an answer to the question, "What are the functions of a stable lipid bilayer structure in living organisms?" Her physics explanation is an answer to the question, "How do lipid molecules self-arrange in water?" Hollis herself does not explicitly identify this distinction, but these questions are of course importantly different and call upon entirely different sets of resources.

The phenomenon itself - the stability of lipid bilayerscan be associated with many different types of questions. Some of these questions call for functional accounts, while others are call for a physical account of how individual molecules form organized structures. Hollis is visibly satisfied that both the physics and biology "explanations" predict the same phenomenological result, the formation of stable lipid bilayers, as evidenced by her fist pump and her assertion that Cindy had "explained it perfectly." Hollis has the expectation not only that both such explanations exist, but that both disciplines ought to play a role in describing the original phenomenon. She expects consistency across disciplinary accounts, and even if different disciplines might ask different questions, she expects that all disciplinary accounts should predict the same behavior.

Whereas the Gavin and Elena examples in Secs. III and IVA, respectively, depict interdisciplinary connections between biochemical rules of thumb and more fundamental physical ideas, the Hollis example illustrates another way in which students cross disciplinary barriers in the IPLS setting. While structure-function relationships are primarily fleshed out in biology classrooms, the inclusion of authentic biological phenomena in IPLS - and encouraging students to draw upon their knowledge of those other disciplines-affords students the opportunity to incorporate both fundamental molecular interactions and structurefunction principles into an interwoven network of resources for thinking about lipid bilayer formation.

\section{THE LANDSCAPE OF INTERDISCIPLINARY CONNECTIONS IN IPLS}

In the preceding episodes we have identified three ways in which students have crossed disciplinary boundaries in meaningful ways (Secs. III-V). We argue in this section that these categories reappear in other instances where students made connections throughout IPLS. We begin by providing two additional examples in which the connections that students make rely on more than one of these basic categories.

The first of these two examples emerged from a discussion of the energy associated with chemical bonds (so-called "chemical energy"). In our course we give explicit attention to the commonly encountered description of the phosphate bond in adenosine triphosphate (ATP) as a "high-energy bond," a phrase used to signify that a large amount of energy can be released when ATP is hydrolyzed in an aqueous medium. The phrase high-energy bond is often incorrectly taken to mean that "bonds store energy," as if there is energy in the bond itself, waiting to escape when the bond is broken $[29,30]$. Of course, this is not the case. Breaking a chemical bond does not release energy; it requires it. ATP can still be considered a high-energy bond, but only if it is understood in the context of an aqueous environment, where bond reformation events do in fact release energy during the cellular process of ATP hydrolysis. Energy is only stored in a bond relative to the energy in the final state of the reaction.

Dreyfus et al. [30] explore how one student in IPLS, Gregor, reconciles his understanding that breaking a chemical bond requires energy with his sense that energy is released during the ATP hydrolysis process. In an interview, Gregor describes how he answered a quiz question related to this apparent contradiction:

I put that when the bond's broken that's energy releasing. Even though I know, if I really think about it, that obviously that's not an energy-releasing mechanism. Because, you can't break a bond and release energy; you always need to put energy in, even if it's a really small amount of energy to break a bond... so I answered that it releases energy, but it releases energy because when an interaction with other molecules, like water, primarily, and then it creates an inorganic phosphate molecule that has a lot of resonance. And is much more stable than the original ATP molecule. So, in the end, it releases a lot of energy..."

Gregor elaborates on this idea, identifying a difference between physics and biology in terms of the questions that each discipline is interested in answering about hydrolysis:

... I've just been taught for a long time that like ATP going to ADP equals like a release of energy... I guess that's just the difference between physics and chemistry and biology. ... Physic[ists] really love to think about things in vacuums, and without context, in a lot of senses. So, you just think about whatever small system you're-isolated system you're looking at, and I guess chemist or biologists thinking about more of an overall context...wherever a reaction or process is happening, that's important to what's going on.

Gregor's statements during this interview indicate that he is drawing on two classes of interdisciplinary connections that we have described: (1) the unpacking of a biochemical heuristic in terms of physical interactions, and (2) the coordination of functional and mechanistic descriptions of biological phenomena. 
Distinguishing the localized ATP bond-breaking event from the concurrent bond reformation events that occur during hydrolysis (and accounting for the energetic changes associated with each individual part of the process) is an example of unpacking a common biological heuristic device in terms of the physical interactions that underlie it. Gregor recognizes that the statement "ATP is a high-energy bond" is a heuristic that must be unpacked in terms of molecular interactions between the ATP molecule and its aqueous environment. The bond-breaking event itself requires energy, while the concurrent bond reformation events with the surrounding water release energy, such that it is possible to see the entire process as one that releases energy even though the ATP molecule itself is stable. The heuristic ATP is a high-energy bond is only sensible when one carefully defines the physical system and considers in detail the physical interactions between the system and its surroundings.

At the same time, Gregor also is coordinating his mechanistic understanding of ATP hydrolysis with a functional understanding of why bond breaking can lead to greater stability in a biological context, and how ATP hydrolysis functions as an energy releasing reaction. He says that energy is released during hydrolysis because it "creates an inorganic phosphate molecule that has a lot of resonance... and is much more stable than the original ATP molecule." Gregor brings chemical stability into the story and points out that, from a biological perspective, ATP bond breaking is meaningful only in the context of the aqueous cellular environment, where ATP has a function. As Gregor puts it, breaking the phosphate bond releases energy only if one considers the "interaction with other molecules, like water." Gregor understands that it makes sense to refer to ATP as a "high-energy bond" only when the molecule is considered in the context of the biological process of ATP hydrolysis.

The interdisciplinary coordination that Gregor articulates in this interview draws on both (1) the unpacking of a biochemical heuristic in terms of physical interactions, and (2) the coordination of functional and mechanistic descriptions of biological phenomena. Function is not as explicit in the Gregor example as it is in Hollis' description of the "biological explanation" for lipid bilayer stability in Sec. V, but it is implicit in his attention to the molecule's function in a biological context.

Gregor is also explicit in his attention to differences in how disciplines bound phenomena. He describes ATP hydrolysis as taking place within an aqueous environment in which a resonance-stabilized molecule forms, and in which the overall reaction (not just the bond breaking event) is important. This points to another type of interaction among the disciplines that is evident in Gregor's comments: attention to system boundaries. In biology, he says, "wherever a reaction or process is happening, that's important to what's going on." To detach a discussion of
ATP hydrolysis from the functional role of ATP in the broader cellular environment (as Gregor sees physicists as being interested in doing) would be to ignore the biological relevance of the hydrolysis process. In this example, a difference in how the disciplines bound phenomena helps account for contradictory ideas: energy input at the bond level, but energy release in the context of coupled reactions. We view attention to system boundaries as a metalevel interaction among disciplines that could apply to any of the examples encountered in those earlier sections. In all those instances, one of the salient features is the importance of understanding where system boundaries lie for different disciplines.

A second example that illustrates the use of multiple classes of interdisciplinary connections can be found in the Violet episode discussed in Sec. IV B. In that discussion we focused on Violet's use of the free energy bridging expression to coordinate her understanding of work as a force applied over a displacement with her understanding of enzymatic catalysis. Like Gregor, however, Violet also alludes to a coordination between functional and mechanistic accounts. In describing how the free energy change $\Delta G$ is relevant for enzymatic activity, Violet says that, "an enzyme is used to catalyze a specific reaction, and that specific reaction will have a $\Delta G$ that corresponds with it." She says that "the reaction does occur 'more fast,' and the enzyme, in order to become active, needs to usually change its conformation."

Violet's descriptions involve terms that indicate she is thinking about the "use" of enzymes and the "need" of enzymes to change conformation in order to carry out its function. These are words related to biological function. Implicitly, Violet is coordinating her functional understanding of enzymatic activity with a mechanistic account of how that activity arises from molecular motions. She does not explicitly label the functional description as "biology" and the mechanistic description as "physics" in the way that Hollis does in Sec. V, but this labeling would be consistent with her descriptions in Sec. IV B.

The Gregor and Violet examples demonstrate how instances of disciplinary boundary crossing in IPLS can incorporate elements from more than one of the three classes of interdisciplinary connections represented in Table I below. The three rows in Table I represent the minimal number of categories needed to account for the space of interdisciplinary connections that we have observed. As mentioned in Sec. II, we came to this classification after conducting 48 (approximately hourlong) interviews with 23 students during the first two year-long iterations of the course, and after filming weekly problem-solving sessions on a weekly basis. Of these 23 students, more than half explicitly referenced episodes that would exemplify one or more of the classes of connections summarized in Table I. Most of these students referenced multiple episodes that would exemplify one or more of the 
TABLE I. Student resources for forming interdisciplinary connections in IPLS are organized into three complementary classes. Each class is a row in the table. The names in parentheses refer to the case study students whose examples were used to exemplify the classes earlier in the paper.

\begin{tabular}{|c|c|c|c|}
\hline & Resource from Physics & & $\begin{array}{l}\text { Resource from } \\
\text { Biology/Chemistry }\end{array}$ \\
\hline 1 & $\begin{array}{l}\text { Forming a physical picture } \\
\text { (or mental simulation) of } \\
\text { microscopic interactions } \\
\text { (e.g., diffusion as the result of } \\
\text { intermolecular collisions and } \\
\text { random walk) }\end{array}$ & $\underset{\text { (Gavin) }}{\longleftrightarrow}$ & $\begin{array}{l}\text { Using a heuristic for predicting } \\
\text { spontaneity (e.g., "particles } \\
\text { know to go from high to low } \\
\text { concentration") }\end{array}$ \\
\hline 2 & $\begin{array}{l}\text { Locating a physical concept } \\
\text { within a bridging equation } \\
\text { (e.g., locating electrostatics or } \\
\text { "work" within the enthalpy term } \\
\text { of the Gibbs free energy } \\
\text { expression) }\end{array}$ & $\begin{array}{c}\longleftrightarrow \\
\text { (Elena/Violet) }\end{array}$ & $\begin{array}{l}\text { Locating a biological or } \\
\text { chemical concept within a } \\
\text { bridging equation (e.g., } \\
\text { associating spontaneity with a } \\
\text { negative change in } \Delta G \text { in the } \\
\text { Gibbs free energy expression) }\end{array}$ \\
\hline 3 & $\begin{array}{l}\text { Explaining a biological } \\
\text { phenomena in terms of } \\
\text { energetic and entropic } \\
\text { contributions } \\
\text { (e.g., lipid bilayer formation as } \\
\text { a result of intermolecular } \\
\text { electrostatic interactions and } \\
\text { molecular degrees of freedom) }\end{array}$ & $\longleftrightarrow$ & $\begin{array}{l}\text { Explaining a biological } \\
\text { phenomena in terms of } \\
\text { function (e.g., lipid bilayer } \\
\text { formation as a result of needing } \\
\text { to transport particular molecules } \\
\text { across a cell membrane) }\end{array}$ \\
\hline
\end{tabular}

three classes. We have yet to identify episodes of interdisciplinary boundary crossing in IPLS that extend beyond some combination of the three classes. That said, our analysis here in no way precludes the possibility that life science students in our course or other IPLS courses might make connections in ways that are more naturally categorized differently.

\section{DISCUSSION}

\section{A. Three classes of interdisciplinary connections}

In the preceding sections we identify three ways in which IPLS students have crossed disciplinary boundaries in meaningful ways: by unpacking biochemical heuristics in terms of underlying physical mechanism (Gavin), by locating both biochemical and physical concepts within a bridging equation (Elena and Violet), and by coordinating functional and mechanistic explanations for the same biological phenomenon (Hollis). These classes are representative of the types of interdisciplinary connections that we have observed over three years of teaching IPLS, as is discussed in Sec. VI. By exploring the Gregor example, we also point to a metalevel interaction that could apply to any of these three classes of gap bridging: the importance of understanding and articulating where system boundaries lie for different disciplines.

It is also possible to think of these classes more abstractly (without the context of any particular phenomenon) by viewing them as coordinating students' conceptual and epistemological resources from physics with those from biology or chemistry. Table I illustrates the resources that we see our students drawing on when making connections in our course.

As we describe in Sec. IV, row 2 of Table I refers to those situations in which a mathematical expression (such as that for Gibbs free energy) serves as a focus at which students can link up their conceptual understanding from physics with their prior coursework in biology and chemistry. This sort of connection, unlike the connection identified in row 1 , does not require that a student have a detailed physical picture of every interaction involved in a process. Understanding where both physical and biological ideas "live" within the single mathematical expression is itself an instance of meaningful boundary crossing.

Not all mathematical relationships are created equal in their capacity to bridge physical and biological ideas. The Gibbs free energy relationship is an especially useful link between fundamental physical and biochemical ideas. This is partly due to the fact that the Gibbs free energy relationship can be seen as a proxy for the second law of thermodynamics [20,30], and that thermodynamics in general is a rich domain for interdisciplinary reasoning [31]. More specifically, life science students are comfortable associating Gibbs free energy with biochemical spontaneity [20], while the enthalpy term $\Delta H$ contains within it internal energy and therefore all the forces typically discussed in introductory physics. In this way, the Gibbs free energy expression is an ideal link between 
biological and physical domains. Locating electrostatics or "work" within the enthalpy term requires, however, that one cross boundaries in which those topics are typically contained.

Row 3 of Table I represents the idea that a student's overall understanding of a biological phenomenon is strengthened when he or she can coordinate between functional and mechanistic stories. Here, too, one's ability to leverage this connection requires a willingness in physics instructors to go beyond canonical conceptual and epistemological boundaries. A physics instructor need not place explicit emphasis on evolution and adaptive advantage to appreciate the added value that functional stories can provide. Affording life science students opportunities to consider biological function alongside physical mechanism helps them to strengthen the interdisciplinary explanatory fabric that supports their understanding of biological phenomena.

A potential metalevel consideration that is connected to each of the classes in Table I is evident in Gregor's awareness of how physics and biology might differently bound the discussion of ATP hydrolysis. The Gregor example illustrates a situation in which an awareness of system boundaries can help locate and potentially resolve disciplinary inconsistencies. In principle, that sort of understanding of disciplinary boundaries could be layered on top of each of the categories of boundary crossing represented in Table I.

\section{B. Mechanism in interdisciplinary sense making}

Implicit in the analysis throughout this paper is the role of physical mechanism in interdisciplinary sense making across biology, chemistry, and physics. It is worth taking a moment to articulate that role more explicitly, to identify the particular way in which physical mechanism appears in each of the classes of interdisciplinary boundary crossing that we have identified.

The class of connections in row 1 of the table-the unpacking of heuristics in terms of detailed microscopic physical interactions - are most easily recognized as mechanistic because they entail chains of interacting entities. We argue that the second class of connectionsthe use of mathematical expressions to bridge conceptual resources from biology and physics - is also mechanistic in nature, even when the analysis does not lead to a full picture of the microscopic physical interactions responsible for the phenomenon. In doing so, we adopt the idea that physical mechanism is characterized by the breaking apart of a complex phenomenon into component parts, and the explanations can black-box the inner workings of some of these components as appropriate [32].

Section IVA describes the lipid bilayer task, an activity designed to explore the energetic and entropic contributions to the spontaneous formation of cell membranes. In designing the lipid bilayer exercise, our goal is not for students to make direct connections between the biochemical heuristic (like dissolves like) and the most fundamental molecular interactions ("fundamental forces between individual lipid and water molecules"). The steps required to make such a connection would be prohibitively complex and context dependent, depending in subtle ways on temperature, lipid concentration, and the length of the lipid hydrocarbon tails. A physical mechanism for bilayer formation that treats the individual lipid and water molecules as objects in an ornate free-body-diagram problem would require a computational approach, not an analytical one. We nevertheless view the connection that Elena makes in this moment as a meaningful marker of interdisciplinary sense making. Although she does not ultimately reach a description in terms of fundamental forces acting on fundamental particles, Elena's unpacking of the like dissolves like heuristic is mechanistic in the sense that she has described the overall spontaneous process in terms of its energetic and entropic components. This "breaking into parts" of a complex phenomenon, and the chaining of these parts together into a logical argument, is a hallmark of mechanistic reasoning [32].

Elena may not come to a complete understanding of how fundamental electric forces push and pull individual lipid and water molecules into their stable bilayer arrangement. We would not expect her to do so. Since one of the goals in discussing bilayer formation in an IPLS course is to facilitate practice in identifying the qualitative roles played by energy and entropy in determining the spontaneity of authentic biological processes, an analytical approach that models the molecules as rigid, noninteracting spheres (or even as molecules interacting with, say, nearest neighbors) would not be appropriate. Such an idealized approach would render the problem biologically inauthentic and would require computational skills rarely encountered at the introductory level. At the same time, an approach that avoids authentic examples like bilayer formation because full analytic accounts are not practical, would fail to leverage resources that students have for thinking about energetic and entropic effects, and thereby fail to facilitate the meaningful interdisciplinary connection that Elena makes.

The third class of connections described in this paperthe coordination of functional explanations for biological phenomena with ones that unpack the energetic and entropic sources of those phenomena-draws attention to the idea that physical mechanisms can be valuably supplemented by accounts that focus on the evolutionary value of biological structures. Rather than avoiding explanations that rely on evolutionary advantage and functionality, this view of interdisciplinary sense making sees both mechanistic and functional explanations as dual pillars supporting a student's overall understanding of particular biological phenomena.

Finally, Gregor's awareness that disciplines make different choices when bounding systems can help students attend to and coordinate different levels of mechanism. 


\section{Affect from interdisciplinary sense making}

All of the examples presented in this paper involve student satisfaction triggered by moments in which physics is helping a student make sense of phenomena previously encountered in his or her biology or chemistry courses. These are moments in which students have made a connection that crosses disciplinary boundaries. It is not unreasonable to expect that some of this satisfaction is directly related to (and perhaps enhanced by) previous frustration in encountering the disciplines as disconnected. Gavin's reflection on his experiences in biology, for example, is accompanied by markers of frustration. He is exasperated that "in biology we never explain that," where "that" refers to the mechanism underlying a heuristic rule about diffusion. Likewise, we see Elena describe having "two separate thoughts" (one about electrostatics, and one about thermodynamics) between which she does not at first see any obvious bridge. We see Hollis struggle to find a "physics explanation" to accompany her "biology explanation" for membrane formation.

We can view these instances of frustration as potentially useful for locating where students perceive gaps and inconsistencies across their disciplinary coursework. Further, their sense of unease and frustration can motivate the need to seek coherence, resolving in positive affective responses. In this sense, affective experiences that emerge from the intersection of disciplines can be seen as indicating potential areas of disciplinary connection that could be further developed in IPLS courses. These could be areas that are particularly motivating for students to explore as they seem to tap into resources for coherence as well as affective satisfaction that can accompany the resolving of inconsistencies $[15,33,34]$.

\section{CONCLUSION AND IMPLICATIONS FOR IPLS COURSES}

Disciplinary fragmentation is a pervasive reality of schooling from K-12 through university [13,35]. In the context of life science students taking introductory physics, this fragmentation is revealed when students describe physics as detached and unrelated to their studies in biology and chemistry. By providing IPLS students with opportunities to meaningfully relate the fundamental physical principles they were learning to rich examples from biology and chemistry, they were afforded an opportunity to build disciplinary bridges that would not otherwise have been possible.

The three classes of intersection that we observed could serve as productive entry points for inviting connection building across disciplines. However, as Sikorski and Hammer have argued [9], we would also caution against assuming that the specific connections that students built in our IPLS context would necessarily be relevant or interesting in other contexts a priori.

Instead, we argue that students, who traverse various course contexts, could be viewed as having the resources to identify gaps and inconsistencies that are relevant to them. Curriculum designers could then incorporate opportunities for students to reason about those gaps.

In the design of our IPLS course this happened in (at least) two ways. First, the instructor was often attentive and responsive to students' articulations of gaps and inconsistencies. As described in Ref. [30], the discussion about the ATP quiz question arose out of the instructor's consideration of why it was that many students came from their biology and chemistry courses with the view that energy was required to break a chemical bond. Rather than "replacing" this view with a different one, the instructor used the example as an opportunity to explore the question of where different disciplines draw system boundaries.

Second, the curriculum design team incorporated student-identified gaps and inconsistencies into subsequent curricular iterations. For example, Gregor's ideas became the inspiration for an essay question that asked students to do the sort of reconciling that Gregor had done, but in even greater detail. In that way, everyone in the class was asked to grapple with the important boundary issues that Gregor raised.

Both of these responses were made possible by moves to frame the IPLS course as building on students' resources from other disciplines. This suggests that a crucial element in supporting opportunities for disciplinary connection is communicating to students that it is appropriate and valuable to bring ideas from other disciplines into the context of a physics course.

Coherence emerges not only by explaining a lot with a little, but also by connecting multiple explanations into a more integrated framework for understanding phenomena in the natural world. The IPLS course is in many ways an ideal setting to do such work.

\section{ACKNOWLEDGMENTS}

Many thanks to Andy Elby for helping us to make sense of the student ideas described in this paper, and to Abigail Daane and Catherine Crouch for thoughtful feedback about the structure of the paper. The authors also thank the UMCP Physics Education Research Group (PERG) and Biology Education Research Group (BERG) for discussions about students' reasoning about the second law. This work was supported by NSF-TUES DUE 11-22818, and the HHMI NEXUS grant. 
[1] D. A. Donovan, L. J. Atkins, I. Y. Salter, D. J. Gallagher, R. F. Kratz, J. V. Rousseau, and G. D. Nelson, Advantages and challenges of using physics curricula as a model for reforming an undergraduate biology course, Cell Biol. Educ. 12, 215 (2013).

[2] L. M. Hartley, J. Momsen, A. Maskiewicz, and C. D' Avanzo, Energy and matter: Differences in discourse in physical and biological sciences can be confusing for introductory biology students, BioScience 62, 488 (2012).

[3] D. C. Meredith and E. F. Redish, Reinventing physics for life-science majors, Phys. Today 66, 38 (2013).

[4] B. O'Shea, L. Terry, and W. Benenson, From $F=m a$ to flying squirrels: Curricular change in an introductory physics course, Cell Biol. Educ. 12, 230 (2013).

[5] B. W. Dreyfus, J. Gouvea, B. D. Geller, V. Sawtelle, C. Turpen, and E. F. Redish, Chemical energy in an introductory physics course for life science students, Am. J. Phys. 82, 403 (2014).

[6] E. F. Redish, C. Bauer, K. L. Carleton, T. J. Cooke, M. Cooper, C. H. Crouch, B.W. Dreyfus, B. D. Geller, J. Giannini, J. Svoboda Gouvea, M. Klymkowsky, W. Losert, K. Moore, J. Presson, V. Sawtelle, K. Thompson, C. Turpen, and R. Zia, NEXUS/Physics: An interdisciplinary repurposing of physics for biologists, Am. J. Phys. 82, 368 (2014).

[7] M. L. Nagel and B. A. Lindsey, Student use of energy concepts from physics in chemistry courses, Chem. Educ. Res. Pract. 16, 67 (2014).

[8] J. Watkins, J. E. Coffey, E. F. Redish, and T. Cooke, Disciplinary authenticity: Enriching the reforms of introductory physics course for life-science students, Phys. Rev. ST Phys. Educ. Res. 8, 010112 (2012).

[9] T. R. Sikorski and D. Hammer, Looking for coherence in science curriculum, Sci. Educ. 101, 929 (2017).

[10] T. Sikorski, Developing an alternative perspective on coherence seeking in science classrooms, Ph.D. thesis, University of Maryland, College Park, 2012.

[11] K. V. Thompson, J. Chmielewski, M. S. Gaines, C. A. Hrycyna, and W. R. LaCourse, Competency-based reforms of the undergraduate biology curriculum: Integrating the physical and biological sciences, CBE-Life Sci. Educ. 12, 162 (2013).

[12] K. Knorr Cetina, Epistemic Cultures: How the Sciences make Knowledge (Harvard University Press, Cambridge, MA, 1999).

[13] A. Phillips, J. Watkins, and D. Hammer, Problematizing as a scientific endeavor, Phys. Rev. Phys. Educ. Res. 13, 020107 (2017).

[14] R. Stevens, S. Wineburg, L. R. Herrenkohl, and P. Bell, Comparative understanding of school subjects: Past, present, and future, Rev. Educ. Res. 75, 125 (2005).

[15] D. Zhang and J. Shen, Disciplinary foundations for solving interdisciplinary scientific problems, Int. J. Sci. Educ. 37, 2555 (2015).

[16] M. Friedman, Explanation and scientific understanding, J. Philos. 71, 5 (1974).

[17] P. Kitcher, Explanatory unification and the causal structure of the world in Minnesota Studies in the Philosophy of
Science: Scientific Explanation (University of Minnesota Press, Minneapolis, 1989), Vol. XIII, pp. 410-499.

[18] H. De Regt and D. Dieks, A contextual approach to scientific understanding, Synthese 144, 137 (2005).

[19] N. Cartwright. The Dappled Word: A Study of the Boundaries of Science (Cambridge University Press, Cambridge, England, 1999).

[20] B. D. Geller, B. W. Dreyfus, J. Gouvea, V. Sawtelle, C. Turpen, and E. F. Redish, Entropy and spontaneity in an introductory physics course for life science students, Am. J. Phys. 82, 394 (2014).

[21] B. D. Geller, B. W. Dreyfus, J. S. Gouvea, V. Sawtelle, C. Turpen, and E. F. Redish, "Like dissolves like": Unpacking student reasoning about thermodynamic heuristics, in Proceedings of the 2013 Physics Education Research Conference, Portland, OR (AIP, New York, 2014), pp. $157-160$.

[22] I. N. Levine, Physical Chemistry (University of Brooklyn, McGraw Hill, New York, NY, 1978).

[23] V. Otero, S. Pollock, and N. Finkelstein, A physics department's role in preparing physics teachers, Am. J. Phys. 78, 1218 (2010).

[24] E. F. Redish and T. J. Cooke, Learning each other's ropes: Negotiating interdisciplinary authenticity, CBE Life Sci. Educ. 12, 175 (2013).

[25] B. D. Geller, J. Gouvea, V. Sawtelle, and C. Turpen, Sources of affect around interdisciplinary sense making, Int. Conf. Learning Sci. 1, 1142 (2014).

[26] G. E. Nilsson, Respiratory Physiology of Vertebrates (Cambridge University Press, Cambridge, MA, 2010).

[27] J. R. Evans and S. Von Caemerer, Carbon dioxide diffusion inside leaves, Plant Physiol. 110, 339 (1996).

[28] V. Sawtelle and C. Turpen, Leveraging a relationship with biology to expand a relationship with physics, Phys. Rev. ST Phys. Educ. Res. 12, 010136 (2016).

[29] W. C. Galley, Exothermic bond breaking: A persistent misconception, J. Chem. Educ. 81, 523 (2004).

[30] B. W. Dreyfus, V. Sawtelle, C. Turpen, J. Gouvea, and E. F. Redish, Students' reasoning about "high-energy bonds" and ATP: A vision of interdisciplinary education, Phys. Rev. ST Phys. Educ. Res. 10, 010115 (2014).

[31] B. Dreyfus, B. Geller, D. E. Meltzer, and V. Sawtelle, Resource letter TTSM1: Teaching thermodynamics and statistical mechanics in introductory physics, chemistry, and biology, Am. J. Phys. 83, 5 (2015).

[32] R. S. Russ, R. E. Scherr, D. Hammer, and J. Mikeska, Recognizing mechanistic reasoning in student inquiry: A framework for discourse analysis developed from philosophy of science, Sci. Educ. 92, 499 (2008).

[33] B. D. Geller, C. Turpen, and C. H. Crouch, Sources of student engagement in introductory physics for life sciences, Phys. Rev. Phys. Educ. Res. 14, 010118 (2018).

[34] L. Z. Jaber and D. Hammer, Engaging in science: A feeling for the discipline, J. Learn. Sci. 25, 156 (2016).

[35] J. Gouvea, V. Sawtelle, and A. Nair, Epistemological progress in physics and its impact on biology, Phys. Rev. Phys. Educ. Res. 15, 010107 (2019). 